\title{
Prediction of Compressive Strength of Plain Concrete Confined with Ferrocement using Artificial Neural Network (ANN) and Comparison with Existing Mathematical Models
}

\author{
S. U. Khan ${ }^{1, *}$, T. Ayub ${ }^{2}$, S. F. A. Rafeeqi ${ }^{2}$ \\ ${ }^{1}$ Urban \& Infrastructure Engineering, NED University of Engineering \& Technology, Karachi, Pakistan \\ ${ }^{2}$ Civil Engineering Department, NED University of Engineering \& Technology, Karachi, Pakistan \\ *Corresponding author: sadaquat78@ hotmail.com
}

Received December 16, 2012; Revised February 02, 2013; Accepted February 28, 2013

\begin{abstract}
This paper is an extension of the work published in year 2010 in which compressive strength of plain concrete confined with Ferrocement was estimated using mathematical models and compared with 55 experimental results. In this paper, predictive model of compressive strength for plain concrete confined with Ferrocement has been developed by using MATLAB Artificial Neural Network (ANN) simulation. Out of 55, 19 experimental results are selected for training of multilayer feed forward neural network. Comparative analysis of the results showed that compressive strength estimated by ANN predictive model are very close to the experimental results than existing theoretical models.
\end{abstract}

Keywords: compressive strength, confinement, ferrocement, wire-mesh layers, artificial neural network

\section{Introduction}

Artificial Neural Network (ANN) is a sub-domain of artificial intelligence system, which has been used progressively since last few years, to solve wide variety of problems in civil engineering applications [1-8]. This informational system is originated from the field of biology to simulate a biological neural network by interconnecting neurons of a brain; however in 1994, engineering definition of ANN has been defined by as a computational mechanism capable of acquiring and working out mapping from a multivariate space of information to another by giving a set of data representing that mapping [9].

The most important property of ANN, which makes it a preferable tool, is its ability of learning directly from examples. Therefore, when rules to solve a problem are not known, or difficult to discover, or conventional computing techniques like regression analysis are very hard to apply, then ANN is very helpful to solve problem [10] as it adapts according to new data and can retrain again; however, it needs sufficient input-output data to include the effect of all parameters [11]. The other important features of ANN include its correct or nearly correct response to incomplete tasks and extraction of information from noisy or poor data; therefore ANN can be applied on an imperfect, fuzzy or strident data due to its learning ability [10]. In Civil Engineering, ANN tool has been applied in various facets such as tidal level forecasting [12], earthquake-induced liquefaction [13], wave-induced seabed instability [14], ultimate bearing capacity of soil [15], prediction of compressive strength [8,11,16-19], slump [20] and workability of concrete [21] etc., and has been found very promising tool.

Multilayer feed forward with back-propagation is the mostly applied network technique in civil engineering and using this technique, slump of ready mixed concrete and strength of concrete has been predicted [21]. Moreover, a neural network model has also been developed to predict the workability of concrete incorporating Metakaolin (MK) and fly ash (FA) [1] and about $98 \%$ accuracy has been achieved in prediction of compressive strength of concrete containing Metakaolin and silica fume [17]. Therefore, due to diversity of ANN, it seems that this tool can be applied to other fields in civil engineering as well.

In 2010, one of the authors of current research paper published a paper [22] in which three (03) mathematical models of compressive strength available in literature, had been used to estimate the strength of plain concrete confined with Ferrocement, and mathematical results had been compared with experimental results. However, current paper aims at the development of a predictive model of compressive strength for plain concrete confined with Ferrocement with the help of the data that has been used previously [22] using Matlab Artificial Neural Network (ANN) tool. Results generated by ANN predictive model have been compared with the results of mathematical models and experimental results.

Authors [22] compiled details of the parameters of plain concrete confined with Ferrocement after referring four (04) studies $[23,24,25,26]$. Details of the parameters has been mentioned in Table 1 which includes cylinder and 
core dimension, no. of Ferro-mesh layers, wire diameter and spacing, yield strength of the wire of Ferro-mesh and unconfined compressive strength. Three types of confinement of Ferrocement has been provided by the authors to the plain concrete $[23,24,25,26]$, which includes Integrally cast Ferro-mesh layers [23,24,25], Ferro-mesh layer in precast shell [23] and Wrapped Ferro-mesh layer on precast core $[23,26]$.

\section{Mathematical Models of Compressive Strength of Plain Concrete Confined with Ferrocement and Their Results}

Till date, three theoretical equations for compressive strength of plain concrete confined with Ferrocement have been proposed in literature [23,24,25] which have been used to develop in a general form [22] as follows:

$$
f_{c t}=f_{c u}+f_{t}
$$

Where, " $f_{c t}$ " is the theoretical compressive strength of concrete confined with Ferrocement. " $f_{c u}$ " is the unconfined compressive strength (i.e. cylinder strength) of plain concrete determined after testing and " $f$ " is the tensile pressure caused by Ferro-mesh layers due to confinement. Theoretical equations proposed by the researchers [23,24,25] has been defined and elaborated underneath:

\subsection{Mathematical Model Proposed by Waliuddin and Rafeeqi [23]}

Waliuddin and Rafeeqi proposed following equation for the strength of concrete confined with Ferrocement:

$$
f_{c t}=f_{c u}+K f_{y}
$$

Where,

$$
K=K_{m} K_{g} K_{p}
$$

Values of $\mathrm{K}_{\mathrm{m}}$ depends on the method of attachment of Ferrocement. For integrally cast Ferro-mesh layers, value of $\mathrm{K}_{\mathrm{m}}$ has been suggested as " 1 "; however, for wrapped Ferro-mesh layers impregnated with mortar and for already cast shell with Ferro-mesh layers, the value of $\mathrm{K}_{\mathrm{m}}$ has been suggested as 0.88 and $0.83 . \mathrm{K}_{\mathrm{g}}$ is the coefficient to account for the grade of concrete and has been suggested by Waliuddin and Rafeeqi as 1, whereas $K_{p}=35 \rho K_{r}$ and $\rho$ is the volume fraction of transverse wires taken over all shell area and $\mathrm{K}_{\mathrm{r}}$ is the ratio of cross sectional to surface area of the shell.

\subsection{Mathematical Model Proposed by Balaguru [24]}

Balaguru proposed following theoretical equation for the confining pressure:

$$
p=\frac{2 R}{d} \operatorname{psi}\left(N / \mathrm{mm}^{2}\right)
$$

Where, "d" is the diameter of cylinder. The confining pressure ( $\mathrm{ft}$ ) is denoted by " $\mathrm{p}$ " in the preceding equation. The ring pressure " $R$ " has been given by following equation:

$$
R=\frac{p}{l} l b / \text { in }(N / m m)
$$

Where "l" is the height of cylinder.

The force " $\mathrm{P}$ " exerted by the transverse wires is given as

$$
P=A_{S} f_{y}
$$

Where $A_{s}$ is the cross sectional area of all the wires across the height of cylinder and $f_{y}$ is the yield strength of the wire.

\subsection{Mathematical Model Proposed by Kaushik and Singh [25]}

Kaushik and Singh [25] proposed equation for $\mathrm{P}_{\mathrm{M}}$ based on the analytical model of Mander et al. [27] to evaluate the strength of axially loaded cylinders confined with Ferrocement and/or containing longitudinal reinforcement. The equation is:

$$
P_{M}=\pi\left(R^{2} \sigma_{0}+R_{M} K_{1} V_{f}\left(R-R_{C}\right) \cdot \frac{w_{y s}}{2}+A_{s t}\left(Y_{s}-\sigma_{c}\right)(7)\right.
$$

Where:

$\mathrm{R}=$ Radius of column, $\sigma_{0}$ is the strength of unconfined concrete, $\mathrm{R}_{\mathrm{M}}$ is the radius of Ferro-mesh layer (mean radius), $K_{1}$ is taken as 4.2 as this value was found to be reasonably accurate, $R_{c}=$ is the radius of core concrete, $\mathrm{W}_{\mathrm{ys}}$ is the mean yield stress of single wire, $\mathrm{A}_{\mathrm{st}}$ is the cross sectional area of longitudinal rebar, $\mathrm{Y}_{\mathrm{s}}$ is the yield strength of longitudinal rebar, $\sigma_{c}$ is the strength of confined concrete and $\mathrm{V}_{\mathrm{f}}$ is the volume fraction of mesh in the casing and is given as

$$
V_{f}=2 \pi \cdot w_{r}^{2} \cdot n_{m} /\left(S_{p}\left(R-R_{c}\right)\right)
$$

Where:

$\mathrm{W}_{\mathrm{r}}$ is the radius of the wire of Ferro-mesh, $\mathrm{n}_{\mathrm{m}}$ is the no.s of Ferro-mesh layer and $S_{p}$ is the perimeter of the confined.

Eq. 7 has been used by the authors [22] in following form:

$$
f_{c t}=f_{c u}+P_{M} / A
$$

Where, A is the area of cylinder.

\section{Development o Prediction Model using Matlab Artificial Neural Network (ANN)}

Generally, ANN is comprised of three layers that are input layer, one or more hidden layer and output layer. Layers between input and output layers is called hidden layer as it contains bulk number of hidden processing units [4]. The input layer of neurons gets information from the outside atmosphere and transmits it to the hidden layer of neurons without computation [28,29]. In order to construct predictive model of compressive strength of 
plain concrete confined with Ferrocement, experimental results of nineteen (19) specimens out of total fifty five (55) has been used, as shown in Table 1. Eight (08)

parameters of the data of nineteen (19) specimens has been used as neurons of external input for training using MATLAB ANN system, as shown in Figure 1.

Table 1. Input Data used for Training in Artificial Neural Network (ANN) [after Ref: 23-26]

\begin{tabular}{|c|c|c|c|c|c|c|c|c|c|c|c|}
\hline \multirow{3}{*}{$\begin{array}{l}\text { Specimen } \\
\text { Label }\end{array}$} & \multicolumn{2}{|c|}{$\begin{array}{l}\text { Cylinder } \\
\text { Dimension }\end{array}$} & \multirow{2}{*}{$\begin{array}{c}\begin{array}{c}\text { Core } \\
\text { Dimension }\end{array} \\
H_{c} \\
\end{array}$} & \multirow{2}{*}{\begin{tabular}{|c}
$\begin{array}{c}\text { No. of Mesh } \\
\text { layers }\end{array}$ \\
$N$
\end{tabular}} & \multirow{2}{*}{$\begin{array}{c}\text { Yield } \\
\text { strength } \\
f_{y}\end{array}$} & \multirow{2}{*}{$\begin{array}{c}\text { Wire } \\
\text { Diameter } \\
d_{w}\end{array}$} & \multirow{2}{*}{$\begin{array}{c}\text { Wire } \\
\text { Spacing } \\
S\end{array}$} & \multirow{2}{*}{$\begin{array}{c}\text { Unconfined } \\
\text { Compressive } \\
\text { Strength }\end{array}$} & \multirow{2}{*}{$\begin{array}{c}\text { Experimental } \\
\text { Confined } \\
\text { Compressive } \\
\text { Strength }\end{array}$} & \multirow{3}{*}{$\begin{array}{l}\text { Method of attachment } \\
\text { of Ferro-mesh layer }\end{array}$} & \multirow{3}{*}{ 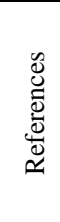 } \\
\hline & $H$ & $D$ & & & & & & & & & \\
\hline & $\mathrm{mm}$ & $\mathrm{mm}$ & $\mathrm{mm}$ & - & $\mathrm{MPa}$ & $\mathrm{mm}$ & $\mathrm{mm}$ & $\mathrm{MPa}$ & $\mathrm{MPa}$ & & \\
\hline Aa-ii-1 & 152 & 305 & 114 & 1 & 345 & 0.96 & 6 & 27.62 & 30.8 & \multirow{5}{*}{$\begin{array}{l}\text { Integrally cast mesh } \\
\text { layers }\end{array}$} & \multirow{10}{*}{ [23] } \\
\hline Aa-ii-3 & 152 & 305 & 114 & 3 & 345 & 0.96 & 6 & 27.62 & 37.52 & & \\
\hline Aa-iii-2 & 152 & 305 & 114 & 2 & 345 & 0.96 & 6 & 26.37 & 33 & & \\
\hline$A b-i-1$ & 152 & 305 & 114 & 1 & 345 & 0.96 & 6 & 32.14 & 35.37 & & \\
\hline$A b-i-3$ & 152 & 305 & 114 & 3 & 345 & 0.96 & 6 & 32.14 & 41.42 & & \\
\hline Ca-iii-1 & 152 & 305 & 114 & 1 & 345 & 0.96 & 6 & 26.37 & 29.31 & \multirow{5}{*}{$\begin{array}{l}\text { Wrapped mesh layer on } \\
\text { precast core }\end{array}$} & \\
\hline Ca-iii-3 & 152 & 305 & 114 & 3 & 345 & 0.96 & 6 & 26.37 & 34.97 & & \\
\hline $\mathrm{Cb}-\mathrm{i}-2$ & 152 & 305 & 114 & 2 & 345 & 0.96 & 6 & 32.14 & 40.29 & & \\
\hline Cb-ii-1 & 152 & 305 & 114 & 1 & 345 & 0.96 & 6 & 30.73 & 33.56 & & \\
\hline Cb-ii-3 & 152 & 305 & 114 & 3 & 345 & 0.96 & 6 & 30.73 & 38.93 & & \\
\hline PB-1 & 150 & 300 & 120 & 1 & 585 & 1.09 & 12.5 & 37.895 & 42.37 & \multirow{3}{*}{$\begin{array}{c}\text { Integrally cast mesh } \\
\text { layers }\end{array}$} & \multirow{2}{*}{ [24] } \\
\hline PB-3 & 150 & 300 & 120 & 3 & 585 & 1.09 & 12.5 & 37.895 & 52.364 & & \\
\hline SKSP-1 & 150 & 300 & 120 & 1 & 340 & 0.7 & 6 & 26.65 & 46.5 & & [25] \\
\hline A2-2 & 150 & 300 & 126 & 2 & 530 & 0.94 & 11.6 & 41.13 & 50.76 & \multirow{3}{*}{$\begin{array}{l}\text { Wrapped mesh layers } \\
\text { on precast core using } \\
\text { special fasteners }\end{array}$} & \multirow{6}{*}{ [26] } \\
\hline A2-4 & 150 & 300 & 114 & 4 & 530 & 0.94 & 11.6 & 41.13 & 48.82 & & \\
\hline A $2-8$ & 150 & 300 & 105 & 8 & 530 & 0.94 & 11.6 & 41.13 & 55.99 & & \\
\hline B2-2 & 150 & 300 & 126 & 2 & 530 & 0.94 & 11.6 & 41.13 & 53.3 & \multirow{2}{*}{$\begin{array}{c}\text { wrapped mesh layers } \\
\text { on precast } \\
\text { core bonded on the } \\
\text { edges }\end{array}$} & \\
\hline B1-8 & 150 & 300 & 105 & 8 & 530 & 0.94 & 11.6 & 41.13 & 60.3 & & \\
\hline C1-8 & 150 & 300 & 105 & 8 & 530 & 0.94 & 11.6 & 41.13 & 71.78 & $\begin{array}{l}\text { Wrapped mesh layers } \\
\text { on precast core by } \\
\text { bonding first two layers }\end{array}$ & \\
\hline
\end{tabular}

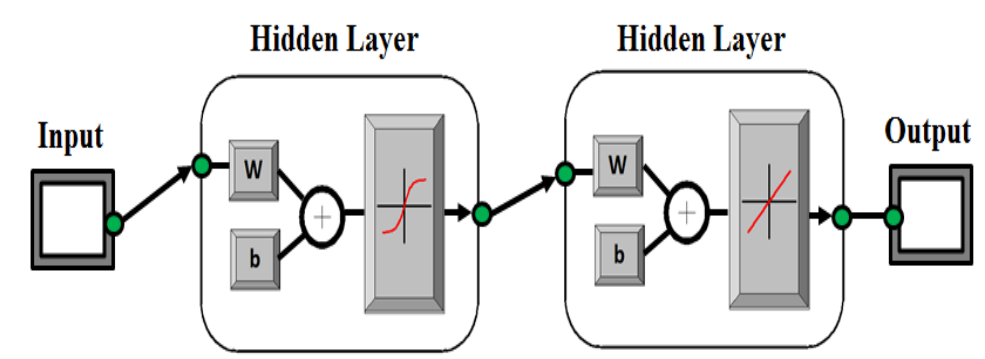
Algorithms
Training:
Performance:
Levenberg-Marquardt (trainlm)
Data Division
Mean Squared Error (mse)
Figure 1. Architecture of the ANN Model Used

Hidden layer has been assigned sixteen (16) neurons, which initially computed linear combination of the output of the neurons of input layer and bias whose coefficients are called "weights" (i.e. coefficients of the linear combination plus bias). After linear combination, neurons in the hidden layer computed by a nonlinear function called "sigmoid function" [3] of the input. Neurons gather information from other neuron through multiplication of the output of connected neuron depending on the synaptic strength of the connection between them [30].

The output of neurons is connected to the network input through a transformation function called activation function [30]. The architecture of ANN used in current paper is comprised of multilayer feed forward and back propagation algorithm. Two-layer tansig/purelin network has been used to construct the model as shown in Figure 1 and tan-sigmoid (tansig) function has been used as transfer function. After initializing the network weights and biases, network was trained for pattern recognition using Levenberg-Marquardt (trainlm) training function. Network inputs and target outputs have been provided for training process. During training, network weights and biases has been iteratively adjusted in order to minimize the network performance function net.performFcn. The default performance function for feed forward networks is mean square error (mse) which is the average squared error between network outputs and the target outputs [31]. The parametric values used in the ANN model are summarized in Table 2.

After training the model with nineteen (19) experimental results, overall data of fifty five (55) specimens has been used as an experimental input for 
simulation which produced similar results to the experimental results. Details of the inputs provided to the
ANN, presented by four researchers [23,24,25,26], are given in Table 3.

Table 2. Information of the Parameters used to develop ANN Predictive Model

\begin{tabular}{|c|c|}
\hline Parameters & Values Used \\
\hline Number of input layer neurons & 8 \\
\hline Number of hidden layer neurons & 16 \\
\hline Number of output layer neuron & 1 \\
\hline Max. Number of epochs & 300 \\
\hline Mu & $1 \times 10^{-8}$ \\
\hline Mu decrease factor & 0.1 \\
\hline Mu increase factor & 10 \\
\hline Performance goal & $1 \times 10^{-5}$ \\
\hline Gradient & $7 \times 10^{-11}$ \\
\hline
\end{tabular}

Table 3. Comparison of the results of ANN predictive model and theoretical models [23-26] with experimental results

\begin{tabular}{|c|c|c|c|c|c|c|c|c|c|c|c|c|c|c|c|}
\hline \multirow{3}{*}{ 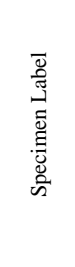 } & \multirow{2}{*}{\multicolumn{2}{|c|}{ 总泀 }} & \multirow{3}{*}{ 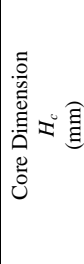 } & \multirow{3}{*}{ 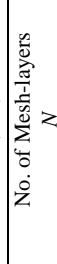 } & \multirow{3}{*}{ 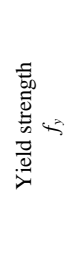 } & \multirow{3}{*}{ 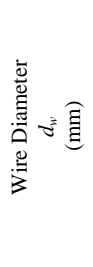 } & \multirow{3}{*}{ 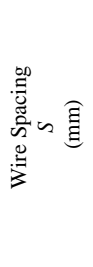 } & \multirow{3}{*}{ 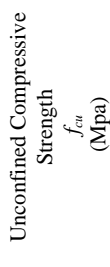 } & \multirow{3}{*}{ 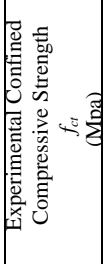 } & \multirow{3}{*}{ 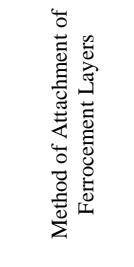 } & \multirow{3}{*}{ 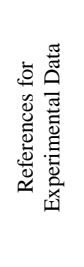 } & \multicolumn{4}{|c|}{$\begin{array}{l}\text { Theoretical Confined Compressive Strength } f_{c t} \\
\text { (Mpa) }\end{array}$} \\
\hline & & & & & & & & & & & & $\bar{v}$ & & & \\
\hline & $H$ & $D$ & & & & & & & & & & 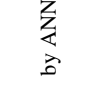 & $\begin{array}{l}0 \\
3 \\
3 \\
3 \\
3\end{array}$ & 西 & 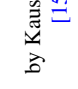 \\
\hline $\begin{array}{l}\text { Aa- } \\
\text { ii-1 }\end{array}$ & 152 & 305 & 114 & 1 & 345 & 0.96 & 6 & 27.62 & 30.8 & \multirow{12}{*}{$\begin{array}{c}\text { Integrally } \\
\text { cast mesh } \\
\text { layers }\end{array}$} & \multirow{17}{*}{ [23] } & 30.25 & 30.88 & 29 & 29.63 \\
\hline $\begin{array}{l}\text { Aa- } \\
\text { ii-2 }\end{array}$ & 152 & 305 & 114 & 2 & 345 & 0.96 & 6 & 27.62 & $\begin{array}{c}34.2 \\
4 \\
\end{array}$ & & & 33.54 & 34.15 & 30.5 & 31.65 \\
\hline $\begin{array}{l}\text { Aa- } \\
\text { ii-3 }\end{array}$ & 152 & 305 & 114 & 3 & 345 & 0.96 & 6 & 27.62 & $\begin{array}{c}37.5 \\
2 \\
\end{array}$ & & & 34.98 & 37.41 & 32 & 33.66 \\
\hline $\begin{array}{l}\text { Aa- } \\
\text { iii-1 }\end{array}$ & 152 & 305 & 114 & 1 & 345 & 0.96 & 6 & 26.37 & 29.6 & & & 28.71 & 29.63 & 27.8 & 28.38 \\
\hline $\begin{array}{l}\text { Aa- } \\
\text { iii-2 } \\
\end{array}$ & 152 & 305 & 114 & 2 & 345 & 0.96 & 6 & 26.37 & 33 & & & 32.43 & 32.9 & 29.3 & 30.4 \\
\hline $\begin{array}{l}\text { Aa- } \\
\text { iii-3 }\end{array}$ & 152 & 305 & 114 & 3 & 345 & 0.96 & 6 & 26.37 & $\begin{array}{c}36.2 \\
2\end{array}$ & & & 34.42 & 36.16 & 30.9 & 32.41 \\
\hline $\begin{array}{c}\mathrm{Ab}-\mathrm{i}- \\
1\end{array}$ & 152 & 305 & 114 & 1 & 345 & 0.96 & 6 & 32.14 & $\begin{array}{c}35.3 \\
7 \\
\end{array}$ & & & 33.66 & 35.4 & 33.4 & 34.15 \\
\hline $\begin{array}{c}\mathrm{Ab}-\mathrm{i}- \\
2\end{array}$ & 152 & 305 & 114 & 2 & 345 & 0.96 & 6 & 32.14 & $\begin{array}{c}38.3 \\
7\end{array}$ & & & 34.86 & 38.67 & 34.8 & 36.17 \\
\hline $\begin{array}{c}\text { Ab-i- } \\
3 \\
\end{array}$ & 152 & 305 & 114 & 3 & 345 & 0.96 & 6 & 32.14 & $\begin{array}{c}41.4 \\
2 \\
\end{array}$ & & & 34.43 & 41.93 & 36.2 & 38.18 \\
\hline $\begin{array}{l}\text { Ab- } \\
\text { ii-1 }\end{array}$ & 152 & 305 & 114 & 1 & 345 & 0.96 & 6 & 30.73 & $\begin{array}{c}33.8 \\
4 \\
\end{array}$ & & & 33.01 & 33.99 & 32.1 & 32.74 \\
\hline $\begin{array}{l}\text { Ab- } \\
\text { ii-2 }\end{array}$ & 152 & 305 & 114 & 2 & 345 & 0.96 & 6 & 30.73 & $\begin{array}{c}36.9 \\
5 \\
\end{array}$ & & & 34.88 & 37.26 & 33.5 & 34.76 \\
\hline $\begin{array}{l}\text { Ab- } \\
\text { ii-3 }\end{array}$ & 152 & 305 & 114 & 3 & 345 & 0.96 & 6 & 30.73 & 40 & & & 34.96 & 40.52 & 34.9 & 36.77 \\
\hline $\begin{array}{c}\text { Ba-ii- } \\
1\end{array}$ & 152 & 305 & 114 & 1 & 345 & 0.96 & 6 & 27.62 & $\begin{array}{c}30.4 \\
5 \\
\end{array}$ & \multirow{5}{*}{$\begin{array}{c}\text { Mesh } \\
\text { layer } \\
\text { in precast } \\
\text { shell }\end{array}$} & & 30.25 & 30.38 & 28.2 & 29.63 \\
\hline $\begin{array}{c}\text { Ba-ii- } \\
2 \\
\end{array}$ & 152 & 305 & 114 & 2 & 345 & 0.96 & 6 & 27.62 & 33.1 & & & 33.54 & 33.16 & 28.81 & 31.65 \\
\hline $\begin{array}{c}\text { Ba-ii- } \\
3 \\
\end{array}$ & 152 & 305 & 114 & 3 & 345 & 0.96 & 6 & 27.62 & $\begin{array}{c}35.8 \\
2\end{array}$ & & & 34.98 & 35.93 & 29.43 & 33.66 \\
\hline $\begin{array}{l}\text { Ba- } \\
\text { iii-1 }\end{array}$ & 152 & 305 & 114 & 1 & 345 & 0.96 & 6 & 26.37 & $\begin{array}{c}29.0 \\
3 \\
\end{array}$ & & & 28.71 & 29.14 & 26.96 & 28.38 \\
\hline $\begin{array}{l}\text { Ba- } \\
\text { iiii-2 } \\
\end{array}$ & 152 & 305 & 114 & 2 & 345 & 0.96 & 6 & 26.37 & $\begin{array}{c}31.8 \\
6 \\
\end{array}$ & & & 32.43 & 31.91 & 27.59 & 30.4 \\
\hline
\end{tabular}




\begin{tabular}{|c|c|c|c|c|c|c|c|c|c|c|c|c|c|c|c|}
\hline $\begin{array}{l}\text { Ba- } \\
\text { iii-3 }\end{array}$ & 152 & 305 & 114 & 3 & 345 & 0.96 & 6 & 26.37 & $\begin{array}{c}34.4 \\
1\end{array}$ & \multirow{7}{*}{$\begin{array}{c}\text { Mesh } \\
\text { layer } \\
\text { in precast } \\
\text { shell }\end{array}$} & \multirow{19}{*}{ [23] } & 34.42 & 34.68 & 28.23 & 32.41 \\
\hline $\begin{array}{c}\text { Bb-i- } \\
1 \\
\end{array}$ & 152 & 305 & 114 & 1 & 345 & 0.96 & 6 & 32.14 & 34.8 & & & 33.66 & 34.91 & 32.68 & 34.15 \\
\hline $\begin{array}{c}\text { Bb-i- } \\
2 \\
\end{array}$ & 152 & 305 & 114 & 2 & 345 & 0.96 & 6 & 32.14 & $\begin{array}{c}37.3 \\
5\end{array}$ & & & 34.86 & 37.68 & 33.24 & 36.17 \\
\hline $\begin{array}{c}\text { Bb-i- } \\
3 \\
\end{array}$ & 152 & 305 & 114 & 3 & 345 & 0.96 & 6 & 32.14 & $\begin{array}{c}39.7 \\
3 \\
\end{array}$ & & & 34.43 & 40.45 & 33.82 & 38.18 \\
\hline $\begin{array}{l}\mathrm{Bb}- \\
\text { ii-1 } \\
\end{array}$ & 152 & 305 & 114 & 1 & 345 & 0.96 & 6 & 30.73 & $\begin{array}{c}33.3 \\
9 \\
\end{array}$ & & & 33.01 & 33.49 & 31.28 & 32.74 \\
\hline $\begin{array}{l}\text { Bb- } \\
\text { ii-2 }\end{array}$ & 152 & 305 & 114 & 2 & 345 & 0.96 & 6 & 30.73 & $\begin{array}{c}35.9 \\
3\end{array}$ & & & 34.88 & 36.27 & 31.86 & 34.76 \\
\hline $\begin{array}{l}\text { Bb- } \\
\text { ii-3 } \\
\end{array}$ & 152 & 305 & 114 & 3 & 345 & 0.96 & 6 & 30.73 & $\begin{array}{c}38.4 \\
8 \\
\end{array}$ & & & 34.96 & 39.04 & 32.45 & 36.77 \\
\hline $\begin{array}{c}\text { Ca-ii- } \\
1\end{array}$ & 152 & 305 & 114 & 1 & 345 & 0.96 & 6 & 27.62 & $\begin{array}{c}30.4 \\
5\end{array}$ & \multirow{12}{*}{$\begin{array}{l}\text { Wrapped } \\
\text { mesh } \\
\text { layer on } \\
\text { precast } \\
\text { core }\end{array}$} & & 30.25 & 30.35 & 28.2 & 29.63 \\
\hline $\begin{array}{c}\text { Ca-ii- } \\
2\end{array}$ & 152 & 305 & 114 & 2 & 345 & 0.96 & 6 & 27.62 & $\begin{array}{c}33.2 \\
7\end{array}$ & & & 33.54 & 33.08 & 28.81 & 31.65 \\
\hline $\begin{array}{c}\text { Ca-ii- } \\
3\end{array}$ & 152 & 305 & 114 & 3 & 345 & 0.96 & 6 & 27.62 & $\begin{array}{c}36.3 \\
9\end{array}$ & & & 34.98 & 35.81 & 29.43 & 33.66 \\
\hline $\begin{array}{l}\text { Ca- } \\
\text { iii-1 }\end{array}$ & 152 & 305 & 114 & 1 & 345 & 0.96 & 6 & 26.37 & $\begin{array}{c}29.3 \\
1\end{array}$ & & & 28.71 & 29.1 & 26.96 & 28.38 \\
\hline $\begin{array}{l}\mathrm{Ca}- \\
\text { iii-2 } \\
\end{array}$ & 152 & 305 & 114 & 2 & 345 & 0.96 & 6 & 26.37 & $\begin{array}{c}32.1 \\
4 \\
\end{array}$ & & & 32.43 & 31.83 & 27.59 & 30.4 \\
\hline $\begin{array}{l}\mathrm{Ca}- \\
\text { iii-3 } \\
\end{array}$ & 152 & 305 & 114 & 3 & 345 & 0.96 & 6 & 26.37 & $\begin{array}{c}34.9 \\
7 \\
\end{array}$ & & & 34.42 & 34.56 & 28.23 & 32.41 \\
\hline $\begin{array}{c}\mathrm{Cb}-\mathrm{i}- \\
1\end{array}$ & 152 & 305 & 114 & 1 & 345 & 0.96 & 6 & 32.14 & $\begin{array}{c}37.6 \\
3\end{array}$ & & & 33.66 & 34.87 & 32.68 & 34.15 \\
\hline $\begin{array}{c}\mathrm{Cb}-\mathrm{i}- \\
2\end{array}$ & 152 & 305 & 114 & 2 & 345 & 0.96 & 6 & 32.14 & $\begin{array}{c}40.2 \\
9\end{array}$ & & & 34.86 & 37.6 & 33.24 & 36.17 \\
\hline $\begin{array}{c}\mathrm{Cb}-\mathrm{i}- \\
3\end{array}$ & 152 & 305 & 114 & 3 & 345 & 0.96 & 6 & 32.14 & $\begin{array}{c}28.7 \\
5\end{array}$ & & & 34.43 & 40.33 & 33.82 & 38.18 \\
\hline $\begin{array}{l}\mathrm{Cb}- \\
\text { ii-1 }\end{array}$ & 152 & 305 & 114 & 1 & 345 & 0.96 & 6 & 30.73 & $\begin{array}{c}33.5 \\
6\end{array}$ & & & 33.01 & 33.46 & 31.28 & 32.74 \\
\hline $\begin{array}{l}\mathrm{Cb}- \\
\text { ii-2 }\end{array}$ & 152 & 305 & 114 & 2 & 345 & 0.96 & 6 & 30.73 & $\begin{array}{c}36.2 \\
2\end{array}$ & & & 34.88 & 36.19 & 31.86 & 34.76 \\
\hline $\begin{array}{l}\text { Cb- } \\
\text { ii-3 } \\
\end{array}$ & 152 & 305 & 114 & 3 & 345 & 0.96 & 6 & 30.73 & $\begin{array}{c}38.9 \\
3 \\
\end{array}$ & & & 34.96 & 38.92 & 32.45 & 36.77 \\
\hline PB-1 & 150 & 300 & 120 & 1 & 585 & 1.09 & 12.5 & 37.895 & $\begin{array}{c}42.3 \\
7\end{array}$ & \multirow{7}{*}{$\begin{array}{c}\text { Integrally } \\
\text { cast mesh } \\
\text { layers }\end{array}$} & \multirow{4}{*}{ [24] } & 41.99 & 42.1 & 42.2 & 43.52 \\
\hline PB-2 & 150 & 300 & 120 & 2 & 585 & 1.09 & 12.5 & 37.895 & $\begin{array}{c}49.6 \\
1 \\
\end{array}$ & & & 45.24 & 46.31 & 46.51 & 49.15 \\
\hline PB-3 & 150 & 300 & 120 & 3 & 585 & 1.09 & 12.5 & 37.895 & $\begin{array}{c}52.3 \\
64\end{array}$ & & & 48.44 & 50.52 & 50.47 & 54.77 \\
\hline PB-4 & 150 & 300 & 120 & 4 & 585 & 1.09 & 12.5 & 37.895 & $\begin{array}{c}54.7 \\
8\end{array}$ & & & 51.64 & 54.73 & 55.12 & 60.4 \\
\hline $\begin{array}{c}\text { SKS } \\
\text { P-1 } \\
\end{array}$ & 150 & 300 & 120 & 1 & 340 & 0.7 & 6 & 26.65 & 46.5 & & \multirow{3}{*}{ [25] } & 46.16 & 28.53 & 27.51 & 27.63 \\
\hline $\begin{array}{c}\text { SKS } \\
\text { P-2 } \\
\end{array}$ & 150 & 300 & 120 & 2 & 340 & 0.7 & 6 & 26.65 & 50 & & & 56.43 & 30.41 & 28.42 & 28.62 \\
\hline $\begin{array}{c}\text { SKS } \\
\text { P-3 }\end{array}$ & 150 & 300 & 120 & 3 & 340 & 0.7 & 6 & 26.65 & 53.5 & & & 66.34 & 32.3 & 29.35 & 29.6 \\
\hline A1-2 & 150 & 300 & 126 & 2 & 530 & 0.94 & 11.6 & 41.13 & $\begin{array}{c}51.3 \\
8 \\
\end{array}$ & \multirow{6}{*}{$\begin{array}{l}\text { Wrapped } \\
\text { mesh on } \\
\text { precast } \\
\text { core by } \\
\text { special } \\
\text { fasteners }\end{array}$} & \multirow{9}{*}{ [26] } & 51.76 & 46.31 & 43.08 & 44.4 \\
\hline A1-4 & 150 & 300 & 114 & 4 & 530 & 0.94 & 11.6 & 41.13 & $\begin{array}{c}52.7 \\
1 \\
\end{array}$ & & & 59.64 & 50.75 & 45.13 & 47.38 \\
\hline A1-8 & 150 & 300 & 105 & 8 & 530 & 0.94 & 11.6 & 41.13 & $\begin{array}{c}50.7 \\
4 \\
\end{array}$ & & & 62.50 & 59.36 & 49.36 & 53.21 \\
\hline A2-2 & 150 & 300 & 126 & 2 & 530 & 0.94 & 11.6 & 41.13 & $\begin{array}{c}50.7 \\
6 \\
\end{array}$ & & & 51.76 & 46.31 & 43.08 & 44.4 \\
\hline A2- 4 & 150 & 300 & 114 & 4 & 530 & 0.94 & 11.6 & 41.13 & $\begin{array}{c}48.8 \\
2\end{array}$ & & & 59.64 & 50.75 & 45.13 & 47.38 \\
\hline A2-8 & 150 & 300 & 105 & 8 & 530 & 0.94 & 11.6 & 41.13 & $\begin{array}{c}55.9 \\
9 \\
\end{array}$ & & & 62.50 & 59.36 & 49.36 & 53.21 \\
\hline B2-2 & 150 & 300 & 126 & 2 & 530 & 0.94 & 11.6 & 41.13 & 53.3 & \multirow{3}{*}{$\begin{array}{l}\text { wrapped } \\
\text { mesh } \\
\text { on } \\
\text { precast } \\
\text { core } \\
\text { bonded } \\
\text { on edgges }\end{array}$} & & 51.76 & 46.31 & 43.08 & 44.4 \\
\hline B1-8 & 150 & 300 & 105 & 8 & 530 & 0.94 & 11.6 & 41.13 & 60.3 & & & 62.50 & 59.36 & 49.36 & 53.21 \\
\hline B1-2 & 150 & 300 & 126 & 2 & 530 & 0.94 & 11.6 & 41.13 & 48.8 & & & 51.76 & 46.31 & 43.08 & 44.4 \\
\hline
\end{tabular}




\begin{tabular}{|c|c|c|c|c|c|c|c|c|c|c|c|c|c|c|c|}
\hline B1-4 & 150 & 300 & 114 & 4 & 530 & 0.94 & 11.6 & 41.13 & $\begin{array}{c}49.5 \\
7\end{array}$ & & & 59.64 & 50.75 & 45.13 & 47.38 \\
\hline C1-4 & 150 & 300 & 114 & 4 & 530 & 0.94 & 11.6 & 41.13 & $\begin{array}{c}59.8 \\
7\end{array}$ & $\begin{array}{l}\text { Wrapped } \\
\text { mesh } \\
\text { layers on } \\
\text { precast } \\
\text { core by } \\
\text { bonding } \\
\text { first two } \\
\text { layers }\end{array}$ & [26] & 59.64 & 50.75 & 45.13 & 47.38 \\
\hline C1-8 & 150 & 300 & 105 & 8 & 530 & 0.94 & 11.6 & 41.13 & $\begin{array}{c}71.7 \\
8\end{array}$ & & & 62.50 & 59.36 & 49.36 & 53.21 \\
\hline
\end{tabular}

\section{Results and Discussion}

Results of compressive strength of plain concrete confined with Ferrocement predicted by ANN model and estimated by existing mathematical models [23,24,25] has been summarised in Table 3. In order to compare the results, Regression value "R", Root mean square error (RMS) and absolute fraction of variance (V) have been calculated from ANN predictive model and existing mathematical models [23,24,25]. The command used in MATLAB for linear regression has been described underneath:

$$
[\mathrm{m}, \mathrm{b}, \mathrm{r}]=\operatorname{postreg}\left(\mathrm{t}_{\mathrm{i}, \mathrm{O}}\right)
$$

Where,

$\mathrm{m}$ is the slope of the linear regression, $\mathrm{b}$ is the $\mathrm{Y}$ intercept of the linear regression, $\mathrm{r}$ is the regression $\mathrm{R}$ value $\left(R=1\right.$ indicates perfect correlation), $t_{i}$ is the Experimental Results (used as target) and $\mathrm{o}_{\mathrm{i}}=$ Theoretical Results (used as output)

Root mean square error (RMS) and absolute fraction of variance (V) has been calculated manually using following formulation:

$$
\begin{gathered}
R M S=\sqrt{\frac{\sum\left|t_{i}-o_{i}\right|^{2}}{n}} \\
V=1-\frac{\sum\left|t_{i}-o_{i}\right|^{2}}{\sum o_{i}^{2}}
\end{gathered}
$$

Where, $\mathrm{n}=$ number of observations

Results of compressive strength of plain concrete confined with Ferrocement predicted by ANN model and estimated by existing mathematical models [23,24,25] has been plotted from Figure 2 to Figure 5 for comparison in terms of closeness to the experimental results. Results showed that ANN model is more efficient than existing mathematical models by comparing the statistical regression value $(\mathrm{R})$, root mean square error (RMS) and absolute fraction of variance $(\mathrm{V})$.

As mentioned by authors [22], mathematical model proposed by Waliuddin and Rafeeqi [23] estimates compressive strength of plain concrete confined with Ferrocement very efficiently and more accurately to the experimental results, than other existing mathematical models, based on all three methods of attachment of Ferro-mesh i.e. integrally cast Ferro-mesh, precast Ferromesh and wrapped Ferro-mesh. However in current research, in spite of using parameters of the data of 19 specimens for training purpose, ANN predictive model exhibited more promising results than all mathematical models $[23,24,25]$.

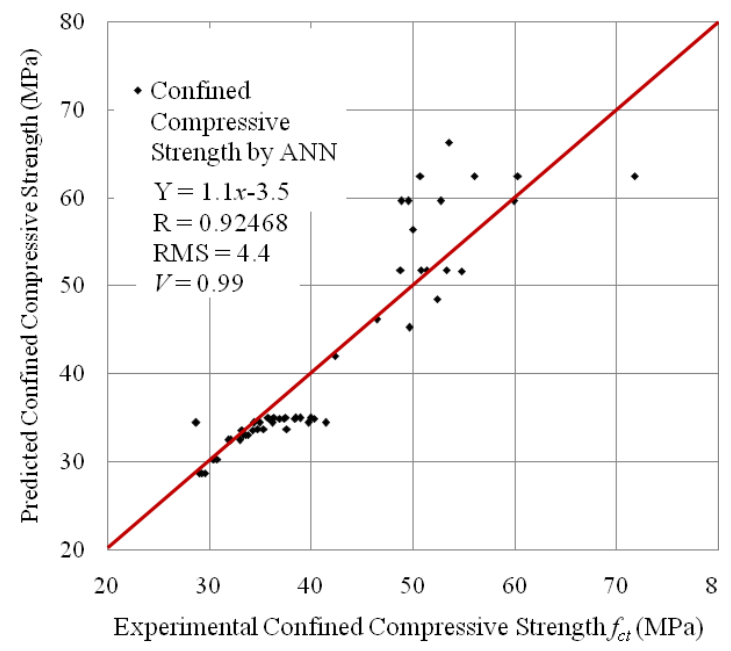

Figure 2. Experimental vs. Predictive ANN Model

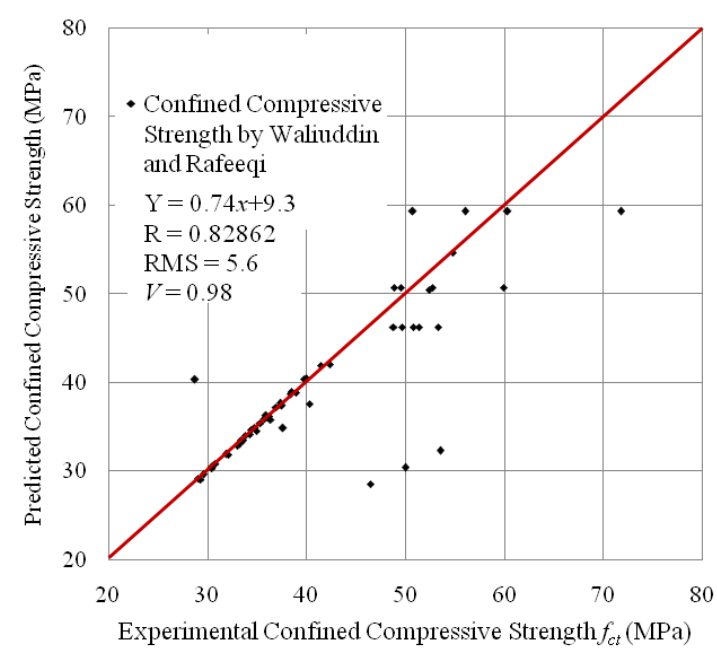

Figure 3. Experimental vs. Theoretical model by Waliuddin and Rafeeqi [15]

\section{Conclusions}

The successful development of the predictive model for the compressive strength of concrete confined with Ferrocement leads to a conclusion that Artificial Neural Network (ANN) is a proficient tool due to its self-learning and generalizing capability from experimental results, as only providing raw information to the training model (i.e. 
cylinder and core dimension, no. of wire-mesh layers, wire diameter and spacing, yield strength of the wire of wire-mesh and unconfined compressive strength), compressive strength of plain concrete confined with Ferrocement has been closed accurately predicted as compared to results of mathematical models [23,24,25].

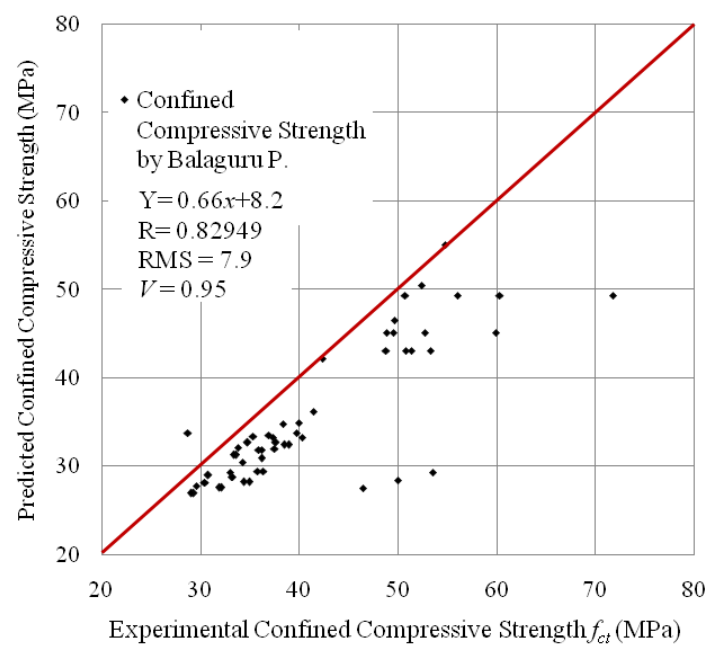

Figure 4. Experimental vs. Theoretical model by Balaguru P. [15]

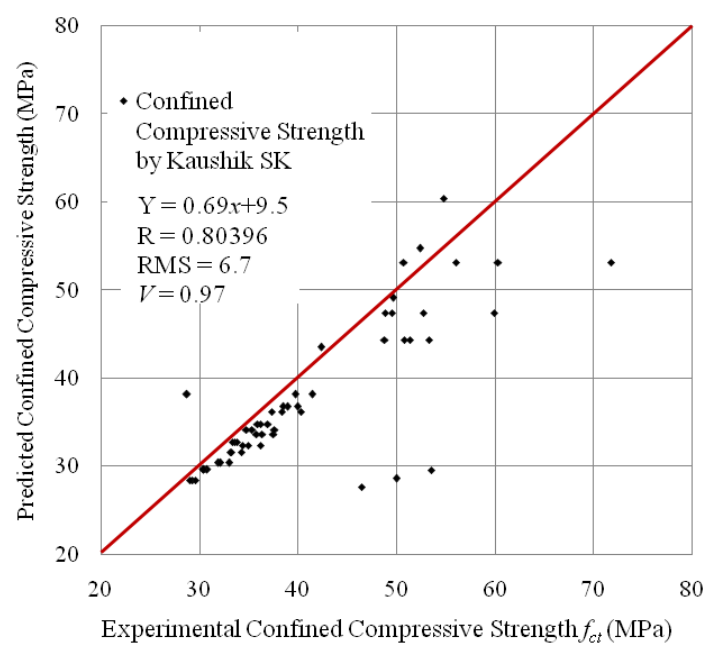

Figure 5. Experimental vs. Theoretical model by Kaushik S.K. [15]

Regression value "R", root mean square error (RMS) and absolute fraction of variance (V) calculated to compare all 55 experimental results [23,24,25,26] with ANN predictive model also shows better performance of ANN predictive model.

Consequently, compressive strength of plain concretes confined with Ferrocement has been predicted in a fairly small period of time with minimal error using multilayer feed forward artificial neural network (ANN) model without performing hectic experimental work. This indicates that multilayer feed forward artificial neural network (ANN) is a feasible method to predict compressive strength of plain concretes confined with Ferrocement.

This study also shows that ANN model as predictive model and model proposed by Waliuddin and Rafeeqi [23] as mathematical model possess capability to predict more accurate compressive strength results for plain concrete confined by different methods such as integrally cast
Ferro-mesh layers, or precast Ferro-mesh layers or wrapped Ferro-mesh layers.

\section{References}

[1] J. Bai, S. Wild, J. Ware, B. Sabir, Using neural networks to predict workability of concrete incorporating metakaolin and fly ash, Adv. Eng. Softw., 34 (2003) 663-669.

[2] R. Ince, Prediction of fracture parameters of concrete by artificial neural networks, Eng. Fract. Mech., 71 (2004) 2143-2159.

[3] B.B. Adhikary, H. Mutsuyoshi, Prediction of shear strength of steel fiber RC beams using neural networks, Constr. Build. Mater. 20 (2006) 801-811.

[4] M.A. Kewalramani, R. Gupta, Concrete compressive strength prediction using ultrasonic pulse velocity through artificial neural networks, Autom. Constr., 15 (2006) 374-379.

[5] M. Pala, E. Özbay, A. Öztaş, M.I. Yuce, Appraisal of long-term effects of fly ash and silica fume on compressive strength of concrete by neural networks, Constr. Build. Mater., 21 (2007) 384-394.

[6] İ.B. Topçu, M. SarIdemir, Prediction of properties of waste AAC aggregate concrete using artificial neural network, Comput. Mater. Sci., 41 (2007) 117-125.

[7] İ.B. Topçu, M. Sarıdemir, Prediction of rubberized concrete properties using artificial neural network and fuzzy logic, Constr. Build. Mater., 22 (2008) 532-540.

[8] I.B. Topcu, M. Saridemir, Prediction of compressive strength of concrete containing fly ash using artificial neural networks and fuzzy logic, Comput. Mater. Sci., 41 (2008) 305-311.

[9] J. Garrett, Where and why artificial neural networks are applicable in civil engineering, J. Comput. Civ. Eng., ASCE, 8 (1994) 129-130.

[10] M. Rafiq, G. Bugmann, D. Easterbrook, Neural network design for engineering applications, Comput. Struct., 79 (2001) 15411552.

[11] S.J.S. Hakim, J. Noorzaei, M. Jaafar, M. Jameel, M. Mohammadhassani, Application of artificial neural networks to predict compressive strength of high strength concrete, Int. J. Phys. Sci., 6 (2011) 975-981.

[12] C.P. Tsai, T.L. Lee, Back-propagation neural network in tidallevel forecasting, J. Waterw. Port Coast. Ocean Eng., 125 (1999) 195.

[13] J. Wang, M. Rahman, A neural network model for liquefactioninduced horizontal ground displacement, Soil Dyn. Earthq. Eng., 18 (1999) 555-568.

[14] D.S. Jeng, D.F. Cha, M. Blumenstein, Neural network model for the prediction of wave-induced liquefaction potential, Ocean. Eng., 31 (2004) 2073-2086.

[15] S. Hakim, S. Jamalaldin, Development and Applications of Artificial Neural Network for Prediction of Ultimate Bearing Capacity of Soil and Compressive Strength of Concrete, in, Universiti Putra Malaysia, (2006).

[16] F. Altun, Ö. Kişi, K. Aydin, Predicting the compressive strength of steel fiber added lightweight concrete using neural network, Comput. Mater. Sci., 42 (2008) 259-265.

[17] M. SarIdemir, Prediction of compressive strength of concretes containing metakaolin and silica fume by artificial neural networks, Adv. eng. softw., 40 (2009) 350-355.

[18] J. Noorzaei, S. Hakim, M. Jaafar, A.A.A. Ali, W. Thanoon, An optimal architecture of artificial neural network for predicting compressive strength of concrete, Indian Concr. J., 81 (2007) 1724.

[19] N. Hong-Guang, W. Ji-Zong, Prediction of compressive strength of concrete by neural networks, Cem. Concr. Res., 30 (2000) 1245-1250.

[20] A. Öztaş, M. Pala, E. Özbay, E. Kanca, N. Çag`lar, M.A. Bhatti, Predicting the compressive strength and slump of high strength concrete using neural network, Constr. Build. Mater., 20 (2006) 769-775.

[21] W. Dias, S. Pooliyadda, Neural networks for predicting properties of concretes with admixtures, Constr. Build. Mater., 15 (2001) 371-379.

[22] S.F.A. Rafeeqi, T. Ayub, Investigation of versatility of theoretical prediction models for plain concrete confined with Ferrocement, Asian J. Civ. Eng., 12 (2010) 337-352. 
[23] A. Waliuddin, S. Rafeeqi, Study of the behavior of plain concrete confined with ferrocement, J. Ferrocem., 24 (1994) 139-151.

[24] P. Balaguru, Use of ferrocement for confinement of concrete, J. Ferrocem., 19 (1989) 135-140.

[25] S. Kaushik, S. Singh, Behavior of Ferrocement Composite Columns in Compression, ACI Spec. Publications, 172 (1997) 669-682.

[26] S.E.M. Mourad, Performance of Plain Concrete Specimens Externally Confined with Welded Wire Fabric, Final research report, King Saud University college of Engineering, (2006).

[27] J. Mander, M.J.N. Priestley, Theoretical stress - strain model for confined concrete, J. Struct. Eng., 114 (1988) 1804.
[28] F. Demir, Prediction of elastic modulus of normal and high strength concrete by artificial neural networks, Constr. Build. Mater., 22 (2008) 1428-1435.

[29] M.Y. Mansour, M. Dicleli, J.Y. Lee, J. Zhang, Predicting the shear strength of reinforced concrete beams using artificial neural networks, Eng. Struct., 26 (2004) 781-799.

[30] A. Mukherjee, S. Nag Biswas, Artificial neural networks in prediction of mechanical behavior of concrete at high temperature, Nucl. Eng. Des., 178 (1997) 1-11.

[31] H. Demuth, M. Beale, Neural network toolbox for use with MATLAB, (1993). 